\title{
From Status Quo Bias to Innovative Multiagent Decisions Under Fuzzy Preferences and Fuzzy Majority
}

\author{
$\operatorname{Janusz~Kacprzyk}^{(\bowtie)}$ \\ Polish Academy of Sciences, Ul. Newelska 6, 01-447 Warsaw, Poland \\ kacprzyk@ibspan.waw.pl
}

\begin{abstract}
Our point od departure is the general problem of multiagent group decision making under fuzzy preferences and a fuzzy majority. We assume a (finite, relatively small) set of options and a set of agents - human beings or software entities, even social groups, if homogeneous - who present their testimonies as fuzzy preference relations over a set of options. We wish to determine an option or a set options that is best acceptable to the group of agents as a whole as to their preferences. This model concerns mainly situations with a relatively small number of options and agents as in the case of human decisions in committees, juries, expert groupl, etc. Assuming Kacprzyk's concept of a fuzzy majority, equated with a fuzzy linguistic quantifiers, exemplified by "most", "almost all", "much more than a half", we use Zadeh's calculus of linguistically quantified propositions, Yager's OWA operators, etc. to derive some group decision solution, notably Kacprzyk's fuzzy Q-cores which is a fuzzy set of options which are not (strongly) defeated in pairwise comparisons (in the sense that other options are preferred over them) by the required fuzzy majority Q, e.g. "most”, of (important) agents. All fuzzy preferences of agents are taken into account and we show that this can be viewed as a reflection of a so called status quo bias which, well known from psychology, which is an emotional bias with a preference for the current state of affairs, the principle of minimal change, etc. A group decision obtained in such a way may be viewed to be non-innovative, and we propose a new approach, with the innovation considered to be an opposite to the status quo. In the new approach, we derive first a social fuzzy preference relation by some aggregation of the individual fuzzy preference relations, that represents the group testimony, which can be viewed as representing some "consensus". Then, for each agent we find a distance of his/her individual fuzzy preference relations to that group (consensory) preference relation which is then used as an additional weight, in addition to the weights corresponding to the relevance of options and importance of individuals. We then argue that a solution (e.g. Q-core) that is best acceptable by, for instance, most agents whose preferences are close to the consensory group fuzzy preference relation, would rather reflect a conservative attitude, while that which is farer from consensory social fuzzy preference relation would rather reflect an innovative attitude. We present some examples of real innovative type decisions using this model.
\end{abstract}

